\title{
Uso del captopril en la crisis hipertensiva inducida por el embarazo
}

\author{
David Vásquez Awad MD*; Fabio Quijano García MD; Mauricio Durán Zamudio MD**
}

RESUMEN: En el Hospital de La Samaritana, durante el período comprendido entre el $1^{\circ}$ de abril de 1992 y el 30 de octubre de 1993 , se tomaron 79 pacientes con Diagnóstico de Pre-eclampsia grave y Eclampsia. 71 de ellos recibieron Captopril sublingüal en dosis de 25 mg cada 30 minutos hasta un máximo de 3 dosis: las 8 restantes recibieron Hidralazina $5 \mathrm{mg}$ IV hasta un máximo de 4 dosis en intervalos de 20 minutos. De las 71 pacientes tratadas con Captopril, 52 (73,3\%) de ellas respondieron exitosamente (disminución de la Presión arterial en $25 \%)$. 20 pacientes $(38 \%)$ requirieron una sola dosis, $26(50 \%)$ requirieron dos dosis y $6(11 \%)$ requirieron 3 dosis. No se observaron efectos adversos importantes en las pacientes ni alteraciones en la diuresis o niveles de creatinina del neonato.

Se recomienda el uso de Captopril sublingüal para el manejo de la Crisis hipertensiva inducida por el embarazo.

PALABRAS CLAVES: Captopril, pre-eclampsia, eclampsia.

SUMMARY: In Hospital de La Samaritana, in the time period between the 1st. of April of 1992 and 30 of October of 1993 , were clasified 79 patients with diagnosis of Severe Pre-eclampsia or Eclampsia. Seventy-one of them recieved 25 mg of sublingüal Captopril every 30 minutes with a maximum of 3 doses. The resting 8 patients recieved Hidralazine $5 \mathrm{mg}$ IV every 30 minutes with a maximum of 4 doses. Of the 71 patients treated with Captopril, $52(73,3 \%$ ) of them responded well (decrease of the Arterial Pressure in $25 \%$ ). Twenty patients (38\%) required one dose, $26(50 \%)$ required 2 doses and $6(11 \%)$ required 3 doses. No important effects were seen in the patients nor alterations in diuresis and creatinina levels in the neonate.

We recommend the use of sublingual Captopril in the management of the Hypertensive Pregnancy Crisis.

KEY WORDS: Captopril, pre-eclampsia, eclampsia.

\section{Introducción}

La HIPERTENSION INDUCIDA POR EL EMBARAZO es un trastorno que ha desafiado a los obstetras de todos los tiempos; como consecuencia de esto se encuentran en la literatura múltiples definiciones y clasificaciones de esta patología. Las decisiones sobre el uso y manejo apropiado de los agentes farmacológicos requieren no sólo de un entendimiento de la fisiopatología de los desórdenes hipertensivos y un reconocimiento de los cambios farmacocinéticos que ocurren durante el embarazo; sino también de una apreciación de los efectos fetales y perinatales de dichos agentes terapéuticos.

El Departamento de Ginecología y Obstetricia del Hospital General Universitario de la Samaritana ha mostrado interés investigativo en el estudio y manejo de los Trastornos Hipertensivos durante el embarazo, situación manifiesta por la realización de los siguientes trabajos:

- Actividad de renina-angiotensina en el embarazo normal y en la HIE (1986) (1).

- Profilaxis de la Hie por medio del uso de Asa (1988) (36).

\footnotetext{
* Instructor Departamento de Ginecología y Obstetricia HGUS.

** Residentes II Departamento de Ginecología y Obstetricia HGUS.
}

- Estudio comparativo entre hidralazina y nifedipina en el manejo de pacientes preeclámpticas y eclámpticas. (1989) (2).

- Estudio comparativo entre sulfato de magnesio y la difenilhidantoína en el manejo de la preeclampsia grave (1990) (3).

- Estudio comparativo entre labetalol e hidralazina en el manejo de la preeclampsia (1991) (4).

- Actividad del sistema del complemento de pacientes con trastornos hipertensivos inducidos por el embarazo (1991) (5).

En el Hospital General Universitario de la Samaritana, se clasifican los trastornos hipertensivos inducidos por el embarazo de acuerdo al Comité de terminología del Colegio Americano de Obstetras y Ginecólogos: (6)

1. Hipertensión crónica

2. Preeclampsia-eclampsia

3. Preeclampsia sobreagregada a enfermedad hipertensiva crónica.

4. Hipertensión gestacional.

5. Trastornos hipertensivos no clasificados.

Los objetivos del tratamiento de la paciente preeclámptica grave y eclámptica se basan en tres ítems fundamentales a saber:

A. Interrupción del embarazo en el momento apropiado y por la vía más segura para el binomio madre-hijo. 
B. Profilaxis y/o tratamiento de las convulsiones, para lo cual existen dos posibilidades terapéuticas:

B.1 Sulfato de magnesio según esquema de Zuspan

B.2 Difenil-Hidantoína según esquema de Ryan.

C. Control del estado hipertensivo, para lo cual el medicamento de primera elección es la hidralazina.

La hipertensión en las pacientes con preeclampsia es la consecuencia de una vasoespasmo a nivel arteriolar. Desde tiempos inmemorables se ha intentado esclarecer la etiología de tal fenómeno patológico para lo cual se ha propuesto innumerables explicaciones; es por esta razón, por lo que se le ha llamado "La enfermedad de las teorías" (8-9).

Está claramente establecido que la HIE se caracteriza por una pérdida de la resistencia vascular a la Angiotensina II. (8).

La mujer embarazada normal muestra un aumento global en el sistema Renina Angiotensina-Aldosterona, pero es refractaria al efecto presor de las sustancias vasoconstrictoras endogenadas como Angiotensina II y Noradrenalina; en cambio, pacientes destinadas a desarrollar HIE muestran una pérdida progresiva de esta refractariedad, haciéndose más sensibles a la vasoconstricción desarrollada por estas sustancias (10-13). De igual manera, se ha visto en pacientes toxémicas un disbalance entre los prostanoides Prostaciclina (PG12) y Tromboxano A2 (TXA2), con marcado incremento de la segunda sustancia que conlleva a vasoconstricción y aumento de la agregación plaquetaria (14-15). El mecanismo fisiopatológico actual de la toxemia incluye los siguientes pasos:

Placentación inadecuada, hipoxia placentaria, peroxidación lipídica placentaria, liberación de radicales libres, agresión endotelial: disbalance de prostanoides + liberación de endotilenas (vasoconstricción renal disparo eje RAA) + disminución de factores relajantes del endotelio (óxido nítrico), vasoconstricción, disminución del flujo útero-placentario, hipoxia placentaria = círculo vicioso (14-19).

LOS INHIBIDORES DE LA ECA tipo CAPTOPRIL, al inhibir el paso de Angiotensina I a Angiotensina II, bloquean la acción vasoconstrictora de esta última, disminuyendo el estímulo a la zona glomerular de la cortical para que no se produzca Aldosterona. Así mismo se bloquea la retención de sodio y agua, ocasionándose mayor diuresis. Por otra parte, actuando sobre el eje Cinina-Calicreina bloquean la Cinasa II, impidiendo la destrucción de Bradicinina, lo que estimula la síntesis de Prostaglandinas vasodilatadoras (19-21). Por otra parte, además de los mecanismos descritos, se ha visto que el Captopril produce una reducción de la Noradrenalina (22), al igual que, debido a la presencia de un grupo sulfidrilo en su estructura, contrarresta la acción de los radicales libres que llevan a la agresión endotelial (23).

Muchos reportes han confirmado la eficacia del Captopril en las Crisis Hipertensivas, en dosis orales de 10 a 50 mgs (24-26). La administración sublingual también ha sido reportada como una exitosa terapia en estas crisis (27-28).
La aparición de la acción del Captopril oral ocurre entre $\operatorname{los} 15$ a 30 minutos posteriores a la ingesta $(24,29)$. La vida sublingual, por su absorción más rápida, que es de 4 a 5 minutos (27), tiene un inicio de acción de los 10 a 20 minutos (28). Aparentemente es efectiva en pacientes con actividad en renina plasmática elevada, como es el caso de las toxémicas (30-31).

Al comparar con otros antihipertensivos se ha visto que el Captopril produce una caída de presión arterial media más paulatina y no decapitante, con un efecto más sostenido $(24-28,31)$.

El uso de Captopril durante el embarazo, de forma aguda, ha sido poco estudiado, pero no se conocen resultados adversos en las pacientes expuestas (7). Su uso crónico se ha asociado en algunos estudios con los siguientes efectos adversos maternos: vértigo, hipotensión, cefalea, fatiga, trastornos del gusto y náuseas $(21,32)$. Los efectos fetales y neonatales reportados son: oligohidramnios, hipotensión fetal prolongada, hipoxia renal y oligoanuria neonatal (33-34). Sin embargo, en la revisión de la literatura se encuentran más de 36 artículos que demuestran ausencia de efectos deletéreos maternofetales $(33,35)$.

Por lo anterior descrito, podría considerarse que el Captopril sublingual que presenta como una alternativa en el manejo agudo de la hipertensión arterial en las pacientes con HIE.

\section{Objetivos y propósitos}

Demostrar la eficacia del Captopril frente a un tratamiento ya establecido como es la Hidralazina, en el manejo de la crisis hipertensiva en la HIE.

\section{Hipótesis básica}

El Captopril sublingual es un medicamento seguro y eficaz para el tratamiento de la Crisis Hipertensiva en la HIE.

\section{Tipo de estudio}

Estudio clínico descriptivo, prospectivo, randomizado, controlado del Departamento de Ginecología y Obstetricia del Hospital General Universitario de la Samaritana.

\section{Materiales y métodos}

El estudio se llevó a cabo en el Departamento de Ginecología y Obstetricia del Hospital General Universitario de la Samaritana (grupo estudio); durante el período de tiempo comprendido entre el $1^{\circ}$ de abril de 1992 y el 30 de octubre de 1993.

Se incluyeron en el estudio todas las pacientes con el diagnóstico de Preeclampsia grave o Eclampsia pura o sobreagregada a EVHC, teniendo en cuenta que no hayan recibido terapia antihipertensiva previa y sin contraindicaciones para el uso de Inhibidores de ECA, como es la hipersensibilidad al fármaco. 
Se definió mejoría como una disminución de la tensión arterial media en un $25 \%$ o descenso de la tensión arterial diastólica a cifras menores o iguales a $100 \mathrm{~mm} \mathrm{Hg}$.

Se considera fracaso la persistencia de la tensión arterial diastólica mayor o igual a $110 \mathrm{~mm} \mathrm{Hg} \mathrm{luego} \mathrm{de}$ tres dosis de Captopril o cuatro de Hidralazina.

Hecho el diagnóstico de Preeclampsia grave o Eclampsia se hospitalizó la paciente en el servicio de Alto Riesgo obstétrico. Se canalizó vena periférica y se colocó una infusión endovenosa de 1000 cc de Lactado de Ringer, además se tomaron exámenes paraclínicos (cuadro hemático, VSG, hemoclasificación, serología, glicemia, BUN, creatinina, ácido úrico, TGO, TGP, bilirrubinas, PT, PTT, plaquetas, PDF, fibrinógeno y uroanálisis). Posteriormente, se inició esquema de profilaxis anticonvulsiva con sulfato de magnesio o fenitoína, se cateterizó vejiga para adecuado control de diuresis y catéter venoso central para medición de Presión Venosa Central.

Ante la persistencia de cifras tensionales diastólicas por encima de $110 \mathrm{~mm} \mathrm{Hg}$ se administró Captopril de la siguiente manera:

Aplicación sublingual de 1 tableta de Captopril (25 mgs), dosis que se repetirá cada 30 minutos hasta un máximo de 3 dosis. De no lograr la disminución de la tensión arterial media de un $25 \%$ o descenso de tensión arterial diastólica a valores inferiores o iguales a $110 \mathrm{~mm}$ HG, se consideró fracaso terapéutico y se administró el esquema alterno con Hidralazina, bolo endovenoso de 5 mgs, repitiéndose si es necesario cada 20 minutos hasta un máximo de 4 dosis.

Se realizaron tomas de tensión arterial cada $10 \mathrm{minu-}$ tos luego de administrada la medicación, hasta obtener TAD $110 \mathrm{~mm} \mathrm{Hg}$. Se evaluaron los efectos adversos durante la administración del fármaco, además de evaluar los siguientes parámetros: FC-FR-Diuresis-reflejosPCF-PVC.

Estabilizada la paciente se inició inducción monitorizado o refuerzo con oxitócicos, y se practicó cesárea sólo por indicación obstétrica.

Al recién nacido se le tomaron muestras de cordón al nacer y las 24 horas para creatinina sérica, además de medición de volumen urinario durante el primer día de vida. Se evaluó Apgar al minuto y a los cinco minutos.

En el puerperio inmediato y ante la persistencia de tensiones arteriales mayores o iguales de $110 \mathrm{~mm} \mathrm{Hg}$, se continuó con Captopril oral a dosis de $25 \mathrm{mgs}$ cada 12 horas, para el esquema de Captopril y Prazosín oral en dosis de $1 \mathrm{mgr}$ cada 12 horas para el esquema de Hidralazina.

\section{Análisis de resultados}

Un total de 79 pacientes participaron en el estudio, setenta y una fueron randomizadas en el grupo CAPTOPRIL y ocho en el grupo hidralazina, este grupo se descontinuó por la ausencia de la medicación en el mercado.

El análisis del grupo captopril presentaba las siguientes características maternas generales:

\section{DISTRIBUCION DE FRECUENCIA SEGUN GRAVIDEZ}

$\begin{array}{cccc}\text { Grav. } & \text { Frecuencia } & \% & \% \text { Acumulado } \\ 1 & 36 & 50.7 & 50.7 \\ 2 & 22 & 31.0 & 81.7 \\ 3 & 10 & 14.1 & 95.8 \\ 4 & 2 & 2.8 & 98.6 \\ 5 & 1 & 1 & 100\end{array}$

No se observó por lo tanto una diferencia en la paridad de las pacientes analizadas, 36 primigestantes y 35 multíparas.

Gráfica 1

CAPTOPRIL EN EL MANEJO DE H.I. E. Distribución por Paridad

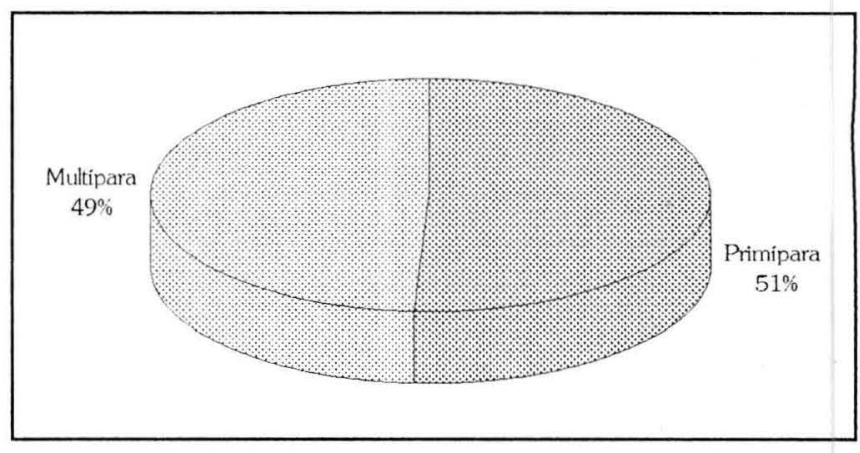

\section{DISTRIBUCION DE FRECUENCIA SEGUN EDAD MATERNA}

Edad Frecuencia \% \% Acumulado

$\begin{array}{lrrr}15-19 & 8 & 11.2 & 11.2 \\ 20-24 & 28 & 39.4 & 50.6 \\ 25-29 & 12 & 16.9 & 67.5 \\ 30-34 & 16 & 22.5 & 90.1 \\ \text { mayor de 35 } & 7 & 9.9 & 100.0\end{array}$

Gráfica 2

CAPTOPRIL EN EL MANEJO DE H.I. E. Distribución por grupos de edad

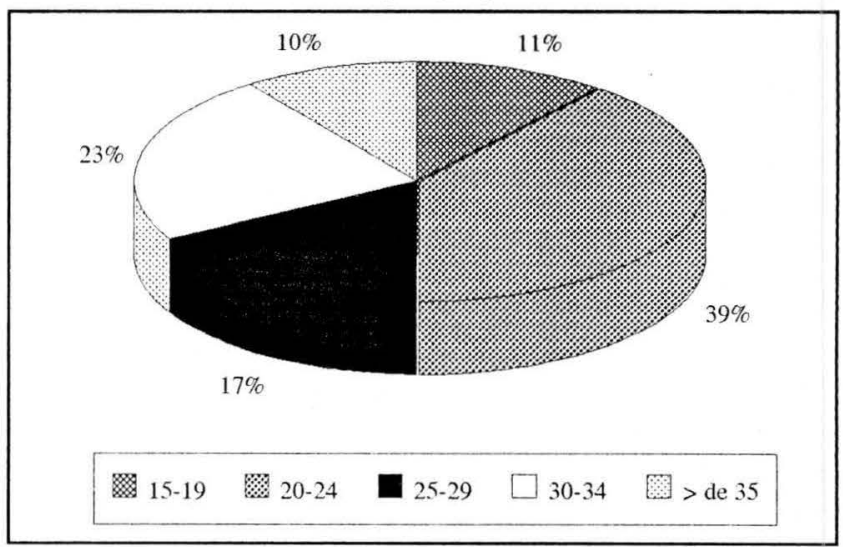


El grupo de edad con mayor frecuencia en nuestro estudio fue el comprendido entre los 20-24 años, el grupo con menor frecuencia estuvo en las pacientes mayores de 35 años.

\section{DISTRIBUCION DE FRECUENCIA SEGUN EDAD GESTACIONAL}

$\begin{array}{lccc}\text { E. Gestacional } & \text { Frecuencia } & \% & \% \text { Acumulado } \\ 20-27 & 8 & 11.2 & 11.2 \\ 28-36 & 32 & 45.0 & 56.2 \\ \text { Término } & 31 & 43.7 & 100.0\end{array}$

Concordantes con los reportes en la literatura el mayor porcentaje de las pacientes a estudio se encontraba en el grupo pretérmino.

\section{Gráfica 3}

CAPTOPRIL EN EL MANEJO DE H.I. E. Distribución por grupos de edad

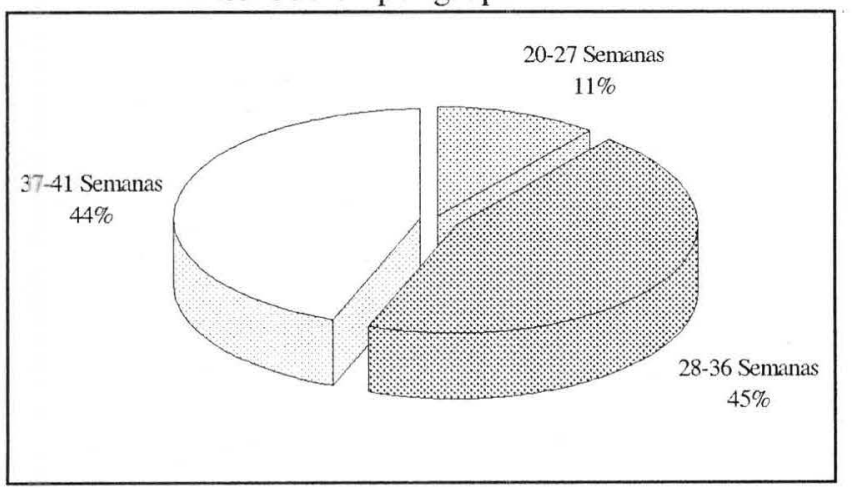

\section{DISTRIBUCION DE FRECUENCIA SEGUN VIA DE NACIMIENTO}

Vía Frecuencia \% \% Acumulado

$\begin{array}{lrrr}\text { Cesárea } & 59 & 83.0 & 83.0 \\ \text { Parto espontáneo } & 4 & 5.6 & 88.6 \\ \text { Parto instrument. } & 8 & 11.4 & 100.0\end{array}$

La principal vía de nacimiento continúa siendo la cesárea en pacientes con preeclampsia grave y eclampsia.

Vale la pena destacar las principales causas de cesárea: de las 59 pacientes que tuvieron esta vía de nacimiento, 40 fueron debidas a un BISHOP desfavorable, 8 pacientes a antecedente de cesárea que no cumplían requisitos para prueba de trabajo de parto, 6 pacientes a sufrimiento fetal agudo, en 3 pacientes la indicación fue Abruptio de placenta y en 2 embarazo gemelar.

De las pacientes que fueron incluidas dentro de la investigación, 35 presentaban control prenatal previo extramural, 22 pacientes no realizaron control prenatal y sólo 14 de las pacientes en el grupo estudiado pertenecían al control prenatal del hospital, curiosamente 8 de estas pacientes recibían asa profiláctica para HIE.
Gráfica 4

CAPTOPRIL EN EL MANEJO DE H.I. E. Vía de Parto

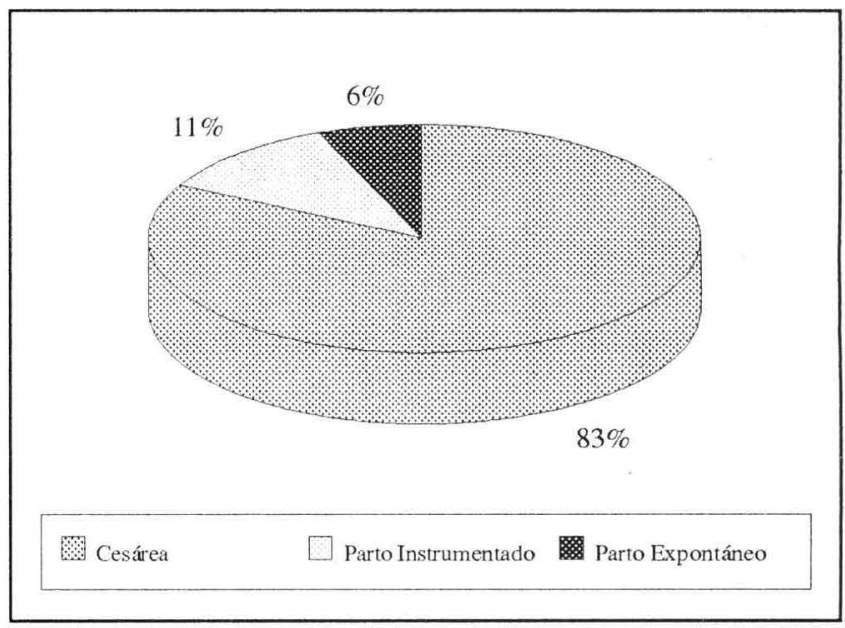

\section{DISTRIBUCION SEGUN DX}

DX Frecuenc. \% \% Acumul.

$\begin{array}{lllr}\text { Preeclampsia grave } & 43 & 60.5 & 60.5 \\ \text { Eclampsia } & 10 & 14.0 & 74.5 \\ \text { EVHC más PE } & 18 & 25.5 & 100.0\end{array}$

Dentro del grupo de las 43 pacientes con diagnóstico de preeclampsia grave, diez ingresaron con diagnóstico de preeclampsia leve y durante la hospitalización desarrollaron un cuadro de grave.

De las 8 pacientes con diagnóstico de eclampsia, 2 ingresaron con diagnóstico de preeclampsia grave y desarrollaron posteriormente una eclampsia, estas pacientes fueron manejadas con sulfato de magnesio como medicación anticonvulsivante inicial.

De las 18 pacientes con diagnóstico de EVHC más PE, 9 ingresaron con TAD mayor o igual a 110 y 9 con TAD menor de 110 y desarrollaron durante la hospitalización cifras mayores o iguales a $100 \mathrm{mmHg}$ de esta manera.

\section{Gráfica 5}

CAPTOPRIL EN EL MANEJO DE H.I. E. Clasificación

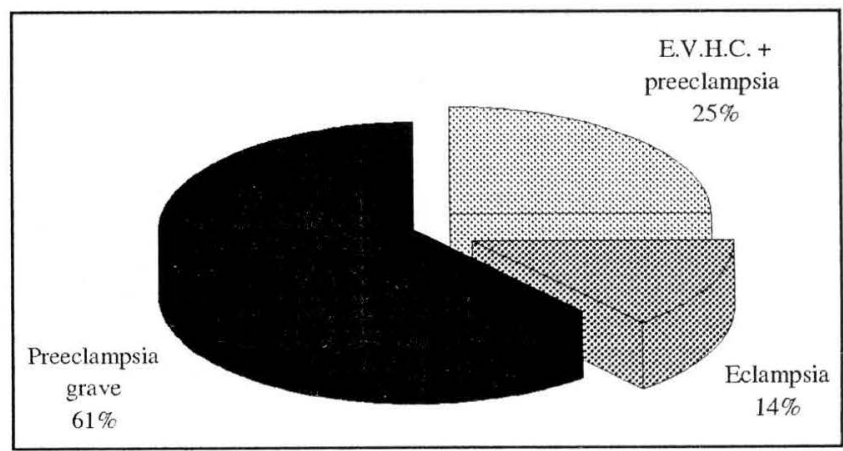


52 pacientes llegaron con $\mathrm{TAD}^{3} 110$.

19 pacientes desarrollaron TAD ${ }^{3} 110$.

\section{DISTRIBUCION DE FCIA SEGUN SINTOMAS}

Síntomas Frecuencia \% \% Acumulado

$\begin{array}{lrrr}\text { 1. } & 28 & 39.4 & 34.4 \\ \text { 2. } & 18 & 25.3 & 64.7 \\ \text { 3. } & 5 & 7.0 & 71.7 \\ \text { 4. } & 20 & 28.3 & 100.0\end{array}$

1. Cefalea; 2. Cefalea + epigastralgia; 3 . Cefalea + epigastralgia + fosfenos; 4 . Ninguno.

Se encontraron los siguientes antecedentes patológicos dentro de las 71 pacientes analizadas: pielonefritis 2; antecedente de HIE - 10; E.V.H.C - 19; úlcera duodenal -1; Sind tónico-clónico generalizado -1 ; trombosis venosa profunda -1; Diabetes -1; Ninguna -36.

El promedio de hospitalización de todo el grupo estudiado fue de 3.8 días; con un mínimo de 2.1 días y un máximo de 12 días. Las complicaciones que se presentaron fueron del $39.4 \%$ distribuidos así: Hellp - 16 pacientes; ACV -2 pacientes; IRA -2 pacientes; endometritis post-parto -2. Se presentaron 2 muertes maternas; una por ACV y otra por Hellp + ACV.

\section{Gráfica 6}

CAPTOPRIL EN EL MANEJO DE H.I. E.

Presentación de hipertension (TAD > $110 \mathrm{mmHg}$ )

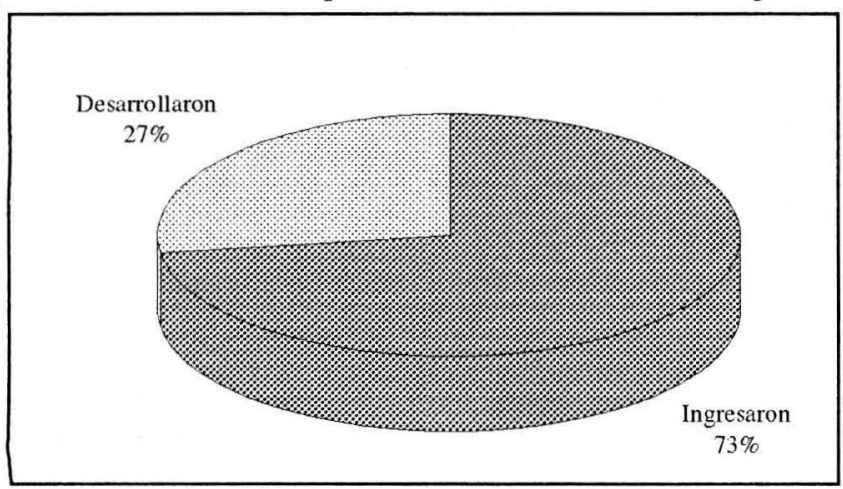

La presión arterial media al ingreso osciló en el rango de $141,5 \mathrm{~mm}$ de $\mathrm{Hg} \pm 15.11 \mathrm{~mm}$ de $\mathrm{Hg}$ y la diastólica en $118 \pm 6 \mathrm{mmHg}$.

La respuesta a la medicación con Captopril sublingual fue la siguiente:

\section{DISTRIBUCION SEGUN NUMERO DE DOSIS}

$$
\text { No. Frecuencia \% \% Acumulado }
$$

$\begin{array}{lrlc}\text { Una dosis } & 20 & 38.46 & 38.46 \\ \text { Dos dosis } & 26 & 50 & 88.46 \\ \text { Tercera dosis } & 6 & 11.6 & 100\end{array}$

\section{Gráfica 7}

CAPTOPRIL EN EL MANEJO DE H.I. E.

Número de dosis requeridas en pacientes con éxito

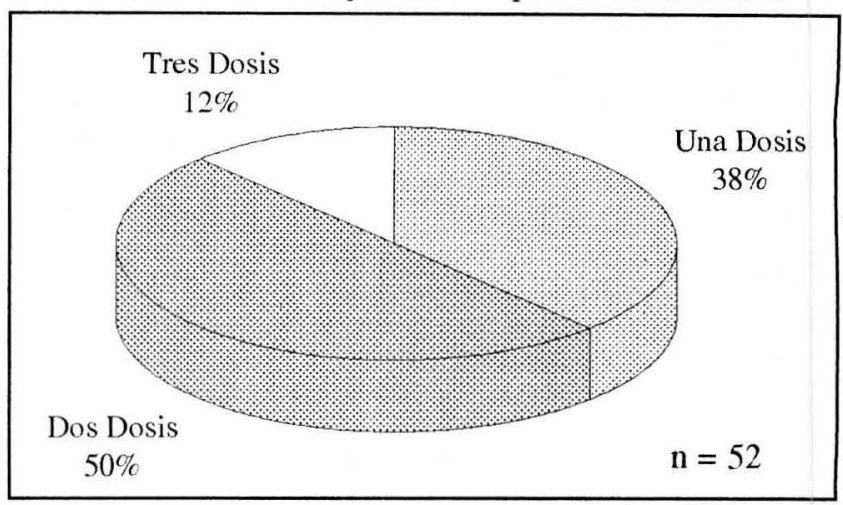

Todas estas 52 pacientes $(73.3 \%)$ consideradas como éxito (Disminución de la TAM en un 25\%). Hubo fracaso a las 19 pacientes restantes $(26.7 \%)$.

De las 19 pacientes consideradas como fracaso; cuatro requirieron hidralazina; nueve nifedipina y 6 pacientes con nitropusiato a dosis de $1.5 \mathrm{ugr} 1 \mathrm{~K} / \mathrm{min}$, con adecuada respuesta.

De las 52 pacientes con las que se obtuvo éxito, se observó un inicio de acción del medicamento a los 12.5 $\pm 3.6 \mathrm{~min}$; logrando una disminución de la P.A.M. de un $26 \%$ a valores de $104 \mathrm{~mm}$ de $\mathrm{Hg}$ y de la P.A.D a valores de $/ 92 \mathrm{~mm}$ de $\mathrm{Hg}$.

\section{Gráfica 8}

CAPTOPRIL EN EL MANEJO DE H.I. E. Resultados de Manejo

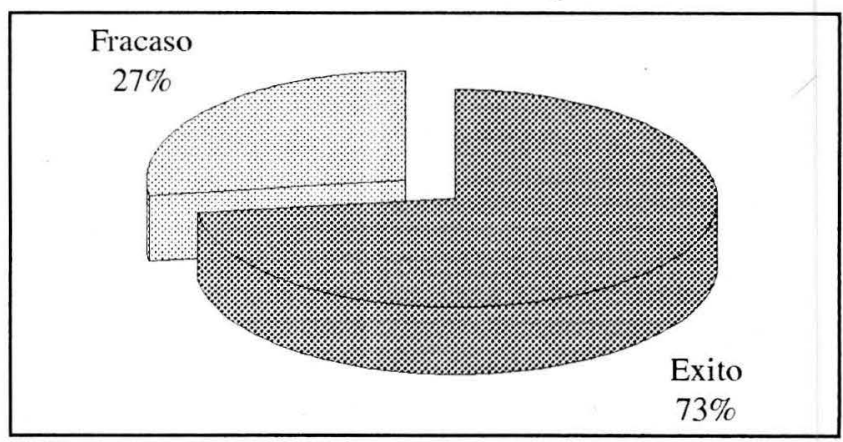

Gráfica 9

CAPTOPRIL EN EL MANEJO DE H.I. E. Dosis Oral Posterior

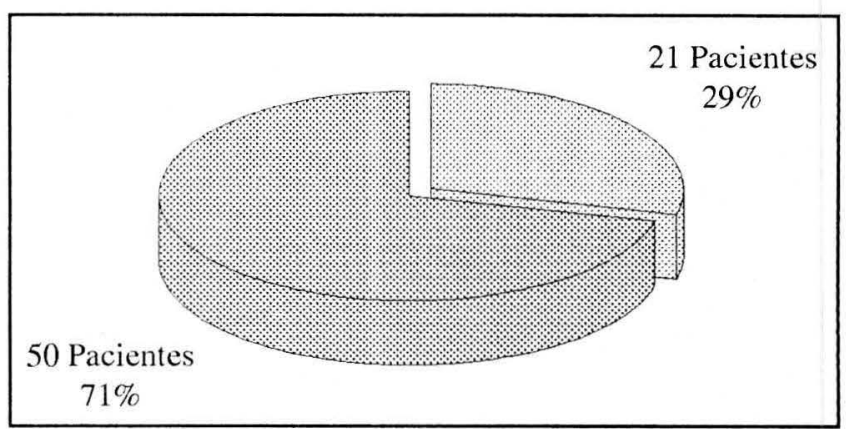


Requirieron dosis oral posterior de $25 \mathrm{mg}$ c/8 horas de captopril 21 pacientes del total de 71 pacientes (29.5\%). De estas 24 pacientes, 18 eran hipertensas crónicas previamente y las 3 pacientes nuevas ingresan con preeclampsia pura; permaneciendo con cifra diastólica ${ }^{3}$ $90 \mathrm{~mm}$ de $\mathrm{Hg}$ una vez cumplido el puerperio.

Dentro de los efectos secundarios del medicamento se hallaron cefalea en 6 pacientes $(9 \%)$; náuseas en 5 pacientes ( $7 \%$ ); fatiga en 2 pacientes $(2.8 \%)$ e hipotensión en 1 paciente $(1.4 \%)$, lo que traduce efectos adversos en un $19 \%$ de los casos.

El análisis de los efectos de la medicación en el RN arrojó los siguientes resultados:

\section{DISTRIBUCION SEGUN APGAR AL MINUTO}

\begin{tabular}{cccc} 
Apgar & Frecuencia & \multicolumn{1}{c}{$\%$} & $\%$ Acumulado \\
2 & 5 & 6.8 & 6.8 \\
3 & 9 & 12.3 & 19.1 \\
6 & 10 & 13.7 & 32.8 \\
7 & 30 & 41.0 & 73.8 \\
8 & 12 & 16.4 & 90.2 \\
9 & 7 & 9.8 & 100.0
\end{tabular}

\section{DISTRIBUCION SEGUN APGAR A LOS 5 MINUTOS}

$\begin{array}{ccrc}\text { Apgar } & \text { Frecuencia } & \% & \% \text { Acumulado } \\ 2 & 3 & 4.1 & 4.1 \\ 4 & 1 & 1.3 & 5.4 \\ 5 & 1 & 1.3 & 6.7 \\ 6 & 5 & 6.8 & 13.5 \\ 7 & 6 & 8.2 & 21.7 \\ 8 & 8 & 10.9 & 32.6 \\ 9 & 21 & 28.8 & 61.4 \\ 10 & 28 & 38.6 & 100\end{array}$

Con respecto a los 73 nacimientos se registró 12 muertes neonatales temprana $(16.4 \%)$ discriminadas por peso de la siguiente manera: ocho neonatos menores de $1000 \mathrm{gm}$ y cuatro menores de $2000 \mathrm{gm}$; la principal causa de muerte fue el bajo peso y el SDR.

Dentro del seguimiento del posible efecto deletéreo del captopril sobre la función renal neonatal encontramos:

\section{DISTRIBUCION SEGUN NIVELES DE CREATININA AL NACER}

\begin{tabular}{ccrc} 
Creatinina & Frecuencia & \multicolumn{1}{c}{$\%$} & \% Acumulado \\
0.8 & 7 & 9.5 & 9.5 \\
0.9 & 3 & 4.1 & 13.6 \\
1.0 & 42 & 57.5 & 71.1 \\
1.1 & 2 & 2.7 & 73.8 \\
1.2 & 11 & 15.0 & 88.8 \\
1.3 & 2 & 2.7 & 91.5 \\
1.4 & 6 & 9.1 & 100.
\end{tabular}

\section{DISTRIBUCION SEGUN NIVELES DE CREATININA A LAS 24 HORAS}

\begin{tabular}{ccrc} 
Creatinina & Frecuencia & \multicolumn{1}{c}{$\%$} & \% Acumulado \\
0.8 & 3 & 5.0 & 5.0 \\
0.9 & 1 & 1.6 & 6.6 \\
1.0 & 31 & 50.8 & 57.4 \\
1.1 & 1 & 1.6 & 59.0 \\
1.2 & 18 & 29.5 & 88.5 \\
1.3 & 4 & 6.5 & 95.0 \\
1.4 & 3 & 5.0 & 100.0
\end{tabular}

Fue evaluada la diuresis de los neonatos durante las primeras 24 horas encontrando promedios de $0.7 \mathrm{cc} / \mathrm{K} /$ hora; ningún recién nacido presentó volúmenes urinarios inferior a $0.5 \mathrm{cc} / \mathrm{K} / \mathrm{h}$ ora.

En cuanto a la profilaxis y/o tratamiento anticonvulsivo se usó indistintamente en 34 pacientes sulfato de magnesio y 47 con Fenitoína.

\section{Discusión}

La hipertensión inducida por el embarazo es una de las principales patologías vistas en nuestro servicio con una incidencia del $33 \%$, constituyendo la principal causa de morbi-mortalidad perinatal.

Uno de los puntos del tratamiento es el control de la TA en pacientes con preeclampsia grave y eclampsia. El antihipertensivo de elección en la hidralazina; fármaco que ha demostrado sus beneficios en múltiples estudios; sin embargo en muchos países incluyendo Colombia esta substancia es de difícil conservación y no está exenta de complicaciones (3-4). Como alternativa dentro del manejo antihipertensivo en nuestro servicio se han utilizado y estudiado algunas medicaciones tales como el Labetalol (4) y Nifedipina (3) sin resultados óptimos.

Como es sabido, el captopril y demás inhibidores de la ECA; están contraindicadas durante la gestación.

En la literatura mundial se reportan múltiples estudios de uso crónico para el manejo de la E.V.H.C durante el embarazo (7, 18-19,33-35), con los conocidos efectos deletéreos sobre el producto.

Están claramente establecidos los beneficios del captopril sublingual en el manejo de las crisis hipertensivas de cualquier etiología; con base en este parámetro, decidimos la utilización de dosis ultracortas cerca del desembarazo como una alternativa a las medicaciones ya conocidas para el manejo de la TA en pacientes con preeclampsia grave o eclampsia.

Se obtuvo un éxito relativo del $72 \%$, lo cual indica que de cada cuatro pacientes manejadas con este fármaco 3 tendrán una adecuada respuesta, sin evidenciar complicaciones maternas, neonatales o fetales atribuidas a la medicación, corroboradas por la medición estricta de creatinina sevia y volúmenes urinarios durante las primeras 24 horas del neonato.

Recomendamos la utilización del captopril sublingual como una eficaz y segura alternativa para el manejo de la crisis hipertensiva en pacientes con preeclampsia grave y eclampsia. 


\section{BIBLIOGRAFIA}

1. Vásquez D. Renina y Angiotensina en el embarazo. 1986.

2. Vásquez D., Cardozo B. Estudio comparativo entre sulfato de magnesio y difenilhidantoína en el manejo de la paciente preeclámptica grave y eclámptica.

3. Vásquez D., Cardozo B., Torres L. Estudio comparativo entre hidralazina eclámptica. 1989.

4. Vásquez D., Cardozo B., Cordova J., Cabrales D., Quijano F. Estudio comparativo entre labetalol e hidralazina en el tratamiento de la preeclampsia grave y eclampsia. 1991.

5. Vásquez D., Bonilla J., Torres L., Durán M. Actividad del sistema de complemento en pacientes con transtornos hipertensivos inducidos por el embarazo. 1991.

6. Hughes EC (ed). Obstet. Gyn. Terminology. Philadelphia: Davis, 1972.

7. Scanferia F., Coli U., Landini S. Treatment of pregnancy induced hypertension with ACE inhibitors enalapril. Clin. Exp. Hypert.1987; 45.

8. Arias F. High risk and delivery. 1989.

9. Creasy R., Resmik R. Maternal-fetal medicine: principles and practice. 1989

10. Chesley LC. Hypertensive disorders in pregnancy. New York: Appleton century crofts. 1978

11. Schwartz R., Retzke U. Cardiovascular response to infusion of angiotensin II in pregnancy women. Obstet. Gyn. 1971; 38: 714.

12. Taliado CE., Chesley Zuspan FP. Renin-angiotensin system in normall and toxemic pregnancies. Differential sensitivity to angiotensin II and norepinephrine in toxemia of pregnancy. Am. J. Obstet. Gyn. 1968; 100: 218.

13. Gant NF., Daley GL. Study of angiotensin II pressor response through out primigravid pregnancy. J. Clin. Invest. 1973; 52: 2682.

14. Furchgott RF. Role of endothelium in response of vascular smooth muscle. Cicres. 1983; 53: 558.

15. Remuzzi G., Marchesi D., Zoja C. Reduced umbilical and placental vascular prostaciclyn in severe preclampsia. Prostaglandine 1980; 20: 105 .

16. Bussolino F., Benedetto C., Massobrio M., Camussi G. Maternal vascular prostaciclyn in preeclampsia. Lancet 1980; 2: 702.

17. DakC., Kefalider A., Sinziger H. Reduced umbilical artery prostaciclyn formation in complicated pregnancy. Am. J. Obstet. Gyn. 1982; 144: 792.

18. Everret RB., Morley RJ., Mcdonald PC. Effect of prostaglandin synthetase inhibitors on pressor response to angiotensin II in human pregnancy. J. Clin. Endocrinol Metab. 1978; 46: 1007.

19. Dusing R., Scherhog R. The converting enzyme inhibitors captopril stimulates prostaciclyn synthesis by insolated rat aorta. Eur. J. Pharm 1983; 91: 501-504.
20. Scherft H., Pretsch R. Converting enzyme inhibitors ramipril stimulates prostaciclyn synthesis by rat aorta: evidence for a kinin-dependente mechanism. Clin wochenschr 1986; 64: 742-745.

21. Jenkin AC., Dreslingkigr R., Gadross SS. Captopril in hypertension seven years later. J. Card Pharm. 1985; 7: 296-301.

22. Xiamg JZ., Linz W., Becker H. Effects on converting enzyme inhibitor ramipril and enalapril on peptide action and sympathetic neurotransmission in the isolated heart. Sur J. Pharmac. 113: 215223.

23. Hestlih H., Mullne K. Does captopril attenuate reperfusion-induced myocardical disfunction by scavenging free radicals? Circulation 1988; 76: 44-51.

24. Case DB., Atlas SA., Sullivan RA. Acute and chronic treatment of severe and malignant hypertension with oral anglotensin-converting enzyme inhibitor captopril. Circulation 1981; 64: 711-765.

25. Biolaz H. Treatment with circulation. 1981; 64: 711-765.

26. Saragoca F. Use of captopril in hypertensive disorders. Lancet 1987; 3: $501-505$.

27. Tschollar G., Davis M. Captopril vs nifedipine in treatment of hypertensive emergencies. New. Engl. J. of Med. 1981; 14: 217-219.

28. Mor J., Merchan A., Calle L. Manejo de urgencias hipertensivas e hipertensión arterial severa con captopril y nifedipina sublingual (estudio preliminar). Clínica Shaio 1989.

29. Ferguson Y., Yenninge A. Sublingual captopril vs nifedipine in hypertensive crises. Lancet 1985; 2: 555.

30. Brunner F. Sublingual captopril in hypertensive crises. Lancet 1985; 2: $34-35$.

31. Dhman KP., Kagedal B., Larsson R., Kalberg B. Pharmacokinetics of captopril and its effects on blood pressure during acute and chronic administration and in relation to food intake. J. Card. Pharmac. 1985; $7(1): 20-24$.

32. Del Portillo H. Hipertensión arterial esencial y nuevos enfoques de fisiopatología y terapéutica. Actas UM. Dpto. M. Interna. 1990.

33. Franz R., Lynn H., Fossum C. Neonatal anuria with maternal angiotensin converting enzime inhibitors. Obstet. Gyn. 1989; 74: 371.

34. Mehta N., Meena M. ACE inhibitor in pregnancy. Lancet. 1989; 196197.

35. Plovin P., Tchobrotsky C. Angiotensin converting enzyme inhibition during human pregnancy. Fefteen cases. Presse med 1985; 14: 2175 2178.

36. Vásquez D., Plata M., Perdomo A. Profilaxis de HIE con ASA. 1988. 